\title{
Spontaneous Orientation of Molecules during Saponification of Poly(vinyl pivalate)
}

\author{
Won Seok Lyoo, Byoung Chul KIM, ${ }^{*, \dagger}$ and Wan Shik HA \\ Department of Fiber and Polymer, Seoul National University, \\ San 56-1, Kwanak, Seoul, Korea \\ * Division of Chemical Engineering, Hanyang University, \\ Haengdang, Seongdong, Seoul 133-791, Korea
}

(Received October 17, 1997)

\begin{abstract}
Two kinds of poly(vinyl alcohol) (PVA) with assigned syndiotactic diad content, 52 to $53 \%$ (denoted as atactic) and 63 to $65 \%$ (denoted as syndiotactic), were prepared by low temperature UV-radiation bulk polymerization of vinyl acetate and vinyl pivalate, followed by saponifying the corresponding poly(vinyl acetate) (PVAc) and poly(vinyl pivalate) (PVPi), respectively. Saponification of PVPi produced highly crystalline and oriented PVA fibers whereas PVAc did not develop any fibrillar structure. The tacticity of the polymer seemed to play a significant role in the in situ fiber formation. Syndiotactic PVA gave positive yield stress while atactic PVA did not exhibit yield behavior. Relaxation time increased with syndiotactic content or molecular weight of polymer. Even by slight shearing between glass plates, syndiotactic PVA solutions readily developed a shear-induced fibrillar structure, while atactic PVA solutions did not exhibit any domain structure. Actual in situ fibrillation at the late stage of saponification of PVPi was simulated by two syndiotactic (PVA)s of similar molecular weight but with different degrees of saponification, 95.4 and $99.0 \%$.

KEY WORDS Poly(vinyl alcohol) / Tacticity / Saponification / In Situ Fibrillation / Rheology / Yield Behavior/
\end{abstract}

Poly(vinyl alcohol) (PVA) is generally prepared by saponifying poly(vinyl ester) precursors such as poly(vinyl acetate) (PVAc) and poly(vinyl pivalate) (PVPi) since vinyl alcohol rearranges to give its tautomer, acetaldehyde, which cannot be polymerized. ${ }^{1}$ Vinyl polymers with different pendant substituents may have different molecular arrangements in space. ${ }^{2} \mathrm{~A}$ polymer in which pendant groups of monomer units are randomly located is called atactic; a polymer in which all the pendant groups are located at the same direction is called isotactic; and a polymer in which the pendant groups are alternately located is called syndiotactic. ${ }^{3}$ The tacticity of a polymer has a profound influence on physical properties. For example, an isotactic polypropylene gives a rigid plastic product whereas an atactic one gives a flexible waxlike one.

Finely fibrillated PVA fibers were formed during saponification of PVPi in the 90/10 (by weight) dimethyl sulfoxide (DMSO)/water with potassium hydroxide. ${ }^{4}$ The in situ fibrillation during saponification was true only for PVPi which has high syndiotactic diad content but not feasible with PVAc having relatively low syndiotactic diad content, suggesting that tacticity played a significant role in the in situ fibrillation process, together with an increase in rigidity of the polymer molecules by changing the chemical structure of the polymer from PVPi to PVA. In this study we traced spontaneous orientation of polymer molecules during saponification of PVPi on rheological bases.

\section{EXPERIMENTAL}

\section{Preparation of Polymer}

Stereoregular polymers are generally prepared through stereospecific polymerization of preordered monomer using a specially designed catalyst. ${ }^{5}$ To obtain poly(vinyl ester) precursors with assigned stereoregularity, vinyl acetate and vinyl pivalate were polymerized by low temperature UV-initiated bulk polymerization. ${ }^{6}$ As predicted, vinyl pivalate produced more stereoregular polymer than vinyl acetate because of steric hindrance of bulky pendent group. The two poly(vinyl ester) precursors were saponified in different ways. In the case of PVAc, $2.5 \mathrm{~mL} 40 \%$ sodium hydroxide aqueous solution was added to a solution of $2 \mathrm{~g}$ PVAc in $100 \mathrm{~mL}$ methanol. ${ }^{7}$ The mixture was stirred for $5 \mathrm{~h}$ at room temperature. The PVA powder thus obtained had syndiotactic content of 52 to $53 \%$, denoted as atactic.

In the case of PVPi, saponification was accomplished using potassium hydroxide. $3 \mathrm{~g}$ PVPi were dissolved in $300 \mathrm{~mL}$ tetrahydrofuran. The solution of potassium hydroxide in the mixture of methanol and water was added to the polymer solution using a dropping funnel with nitrogen flushing. PVA so prepared had syndiotactic diad content of 63 to $65 \%$, denoted as syndiotactic. The saponification of stereoregular PVPi produced a solid fibrillar reaction mixture, which produced highly oriented fibrillated fiber when beaten mechanically or opened on an ultrasonic generator containing a methanol solution. Filtering and washing the fiber bundles yielded bright yellow PVA fibers.

To measure the molecular weight of polymer, PVA was reacetylated as follows. A mixture of $1 \mathrm{~g}$ PVA, $2 \mathrm{~mL}$ pyridine, $20 \mathrm{~mL}$ acetic anhydride, and $20 \mathrm{~mL}$ acetic acid was stirred in nitrogen atmosphere at $100^{\circ} \mathrm{C}$ for $24 \mathrm{~h}$ for atactic PVA and for $72 \mathrm{~h}$ for syndiotactic PVA. ${ }^{8}$ The reacetylated PVAc was dissolved in benzene at $0.5 \mathrm{~g} \mathrm{dl}^{-1}$, and reduced viscosity was measured with an Ubelhode viscometer, diluting step by step to $0.2 \mathrm{~g} \mathrm{dl}^{-1}$ at $30^{\circ} \mathrm{C}$. Intrinsic viscosity $([\eta])$ was determined by extrapolating

† To whom all correspondences should be addressed (Fax. +82-2-297-5859, Tel. +82-2-290-0494). 
reduced viscosity to the zero concentration on the plot of reduced viscosity against polymer concentration. The molecular weight of PVA was calculated by the MarkHouwink equation expressed as ${ }^{9}$

$$
[\eta]=8.91 \times 10^{-3}\left[P_{n}\right]^{0.62}
$$

in which $P_{n}$ is the number-average degree of polymerization of polymer.

\section{Characterization of Molecular Structure of Polymer}

The syndiotactic triad and syndiotactic diad content of PVA was measured using a proton-nuclear magnetic resonance $\left({ }^{1} \mathrm{H}\right.$ NMR) spectrometer, for solutions of PVA in DMSO by Varian Sun Unity 300 NMR spectrometer. The degree of saponification was determined by weight loss after saponification and the ratio of tert-butyl and methylene proton peaks in the ${ }^{1} \mathrm{H}$ NMR spectrum.

\section{Observation of Morphology of Solution and Fibers}

Solutions of PVA in DMSO were prepared by stirring the polymer/DMSO mixture at $45^{\circ} \mathrm{C}$. To exclude the possibility of incomplete dissolution of the polymer in the solvent, the solution was optically examined. With syndiotactic PVA, one week was required to obtain optically homogeneous polymer solution. The phase morphology of PVA solutions was observed by Scalar video microscope VMS-3000 equipped with a Sony color video printer. PVA solution was poured onto a glass plate. The solution layer on the glass plate was made thin enough to transmit the light and a cover-glass was carefully put on the solution layer. To reach a completely relaxed state, the solution sample was placed still without disturbance for $90 \mathrm{~min}$ and unsheared (static-state) morphology was photographed with the VMS-3000 system in a transmission mode. Sheared morphology was observed by video-taping changes in solution layer morphology with time after slightly moving the upper glass plate at the velocity of $c a .2 \mathrm{~mm} \mathrm{~s}^{-1}$ for $0.5 \mathrm{~s}$. The structure of PVA fiber in situ formed during saponification of PVPi was observed by scanning electron microscope, Hitachi S-510.

Measurement of Rheological Properties of PVA Solutions

For more precise rheological measurement, a Rheometrics fluid spectrometer RFS II was used. Minimum sensitivity and sampling time of RFS II are $2 \times 10^{-3} \mathrm{gcm}$ and $5 \mathrm{~ms}$, respectively. In RFS measurement, a coneand-plate type flow cell with diameter of $25 \mathrm{~mm}$ and degree of 0.02 rad was used to effectively accommodate the torque of the solutions. To keep the shear history of polymer solutions constant, the solution was placed without disturbance between the cone and plate for 90 min before rheological measurement.

\section{RESULTS AND DISCUSSION}

For spontaneous in situ fibrillation during saponification of PVPi to PVA, preparation of a high molecular weight poly(vinyl ester) precursors with little branched structures is required. To obtain linear and high molecular weight PVA with assigned tacticity, low temperature $\mathrm{UV}$-initiated bulk polymerization is used in the preparation of the precursor polymers. ${ }^{8,10,11}$ Two precursor polymers with different syndiotactic diad content, PVAc and PVPi, are prepared by low temperature UV-initiated bulk polymerization. Saponification of the precursors produces (PVA)s with assigned stereoregularity as given in Table I.

Saponification of syndiotactic PVPi produces an in situ formed PVA fiber in Figure 1. The PVA fiber thus produced has unique morphology, a laminarly-associated pulp-like fiber structure. This laminated fibrillar structure is totally different from the filament structure, typical of conventional man-made fibers prepared by spinning with subsequent longitudinal drawing. Up to now, the laminar fiber structure has been usually observed in naturally occurring cellulose fibers such as ramie and jute. Such a fiber structure is also reported in other unspun fibers obtained by in situ fibrillation during growth packing polymerization of poly ( $p$-phenylene terephthalamide) and extrusion of hydrated acrylic melts in the supercooled state. $^{12}$

The PVA fiber thus obtained has a highly crystalline and well oriented structure. The dimensions and physical properties of the in situ formed fibers are dependent on saponification conditions and chemical properties of the precursor polymer. ${ }^{13}$ The PVA fiber is very thin, diameter from 1 to $50 \mu \mathrm{m}$, but length up to $30 \mathrm{~cm}$. As a rule, the tenacity of isolated fibril increases with molecular weight of polymer and content of syndiotactic diad. The PVA

Table I. Effects of polymerization conditions on molecular parameters of PVA

\begin{tabular}{|c|c|c|c|c|c|}
\hline \multicolumn{3}{|c|}{ Polymerization conditions } & \multirow{2}{*}{$P_{n}$} & \multirow{2}{*}{$\begin{array}{c}\begin{array}{c}\text { Syndiotactic } \\
\text { diad content }\end{array} \\
\%\end{array}$} & \multirow{2}{*}{$\begin{array}{c}\begin{array}{c}\text { Degree of } \\
\text { saponification }\end{array} \\
\%\end{array}$} \\
\hline Monomer & Type & Temperature & & & \\
\hline Vinyl acetate & Photo-bulk & $10^{\circ} \mathrm{C}$ & 3,100 & 52.7 & $99.0<$ \\
\hline Vinyl acetate & Photo-bulk & $10^{\circ} \mathrm{C}$ & 3,900 & 52.2 & $99.0<$ \\
\hline Vinyl acetate & Photo-bulk & $10^{\circ} \mathrm{C}$ & 4,500 & 52.9 & $99.0<$ \\
\hline Vinyl acetate & Photo-bulk & $10^{\circ} \mathrm{C}$ & 6,100 & 52.3 & $99.0<$ \\
\hline Vinyl pivalate & Photo-bulk & $10^{\circ} \mathrm{C}$ & 6,300 & 63.5 & $99.0<$ \\
\hline Vinyl pivalate & Photo-bulk & $10^{\circ} \mathrm{C}$ & 9,800 & 63.1 & $99.0<$ \\
\hline Vinyl pivalate & Photo-bulk & $10^{\circ} \mathrm{C}$ & 12,300 & 63.8 & $99.0<$ \\
\hline Vinyl pivalate & Photo-bulk & $10^{\circ} \mathrm{C}$ & 15,500 & 63.5 & $99.0<$ \\
\hline Vinyl pivalate & Photo-bulk & $0^{\circ} \mathrm{C}$ & 14,000 & 64.5 & $99.0<$ \\
\hline Vinyl pivalate & Photo-bulk & $0^{\circ} \mathrm{C}$ & 14,300 & 64.5 & $95.4<$ \\
\hline
\end{tabular}

Ultraviolet ray was used as a photo source in polymerization. 
Table II. Effects of the degree of saponification on relaxation behavior of syndiotactic PVA

\begin{tabular}{lccc}
\hline $\begin{array}{c}\text { Relaxation } \\
\text { time }(\lambda)\end{array}$ & PVA1 $^{\mathrm{a}} / \mathrm{s}$ & PVA2 $^{\mathrm{b}} / \mathrm{s}$ & PVA3 $^{\mathrm{c}} / \mathrm{s}$ \\
\hline at $0.2 \mathrm{~s}^{-1}$ & 4.849 & 5.002 & 4.867 \\
at $0.5 \mathrm{~s}^{-1}$ & 2.005 & 2.021 & 1.989 \\
at $1 \mathrm{~s}^{-1}$ & $9.870 \times 10^{-1}$ & 1.001 & $9.756 \times 10^{-1}$ \\
at $5 \mathrm{~s}^{-1}$ & $1.904 \times 10^{-1}$ & $1.971 \times 10^{-1}$ & $1.795 \times 10^{-1}$ \\
at $10 \mathrm{~s}^{-1}$ & $9.325 \times 10^{-2}$ & $9.883 \times 10^{-2}$ & $8.664 \times 10^{-2}$ \\
at $50 \mathrm{~s}^{-1}$ & $1.858 \times 10^{-2}$ & $1.926 \times 10^{-2}$ & $1.702 \times 10^{-2}$ \\
at $100 \mathrm{~s}^{-1}$ & $9.045 \times 10^{-3}$ & $9.634 \times 10^{-3}$ & $8.418 \times 10^{-3}$ \\
\hline
\end{tabular}

${ }^{a} P_{n}, S$-diad, and degree of saponification (D.S.) of PVAl are 15500 , $63.5 \%$, and over $99.0 \%$, respectively. ${ }^{\mathrm{b}} P_{n}, S$-diad, and D.S. of PVA2 are $14000,64.5 \%$, and over $99.0 \%$, respectively. ${ }^{\mathrm{c}} P_{n}, S$-diad, and D.S. of PVA3 are $14300,64.5 \%$, and $95.4 \%$, respectively.
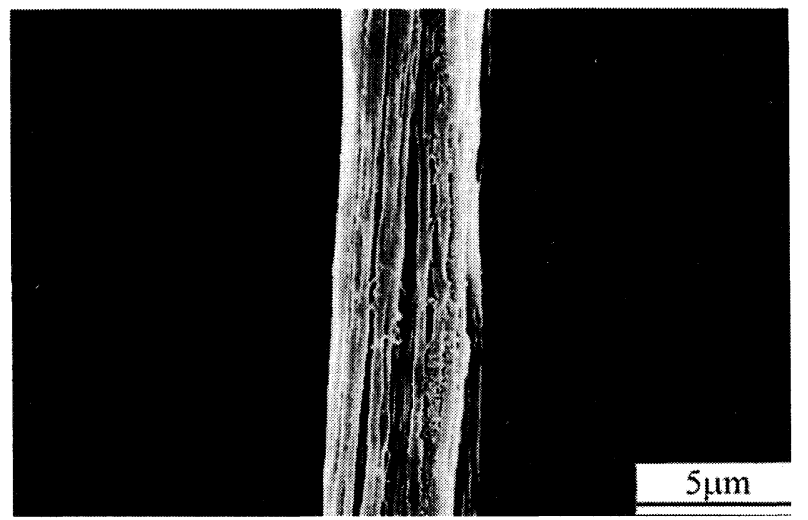

Figure 1. Microstructures of PVA fiber in situ fibrillated during saponifying syndiotactic PVPi.

fiber obtained from PVPi of $P_{n}$ of 17000 has tenacity as high as $16 \mathrm{~g} \mathrm{den}^{-1}$ without any chain orientation by drawing and heat treatment (annealing).

As expected tacticity has a profound influence on the rheological properties of vinyl polymers. The complex viscosity $\left(\eta^{*}\right)$ of $2 \mathrm{wt} \%$ solutions of atactic and syndiotactic (PVA)s of several different molecular weights in DMSO is plotted against loss modulus $\left(G^{\prime \prime}\right)$ at room temperature in Figures 2 and 3, respectively. First, a clear difference in rheological response exists between atactic and syndiotactic (PVA)s. Syndiotactic PVA generally gives much higher viscosity than atactic PVA at the identical polymer concentration. Secondly, atactic PVA solutions give rheological response typically found in solutions of flexible chain polymers, whereas syndiotactic PVA solutions do not give a Newtonian flow region at low frequency as shown in Figure 3. Disappearance of the lower Newtonian flow region indicates heterogeniety of a system frequently observed in inorganic-filled systems, liquid crystalline polymers, and block copolymers. ${ }^{14-17}$

Figures 4 and 5 present plots of $G^{\prime \prime}$ against $\omega$ for $2 \mathrm{wt} \%$ solutions of atactic and syndiotactic (PVA)s of different $\left(P_{n}\right)$ s in DMSO at $30^{\circ} \mathrm{C}$, respectively. With both polymers, PVA of higher molecular weight gives rise to greater $G^{\prime \prime}$ at given frequency. It should be noted that the two polymers show different curve slopes. In the case of atactic PVA, the slope is almost 1 and remains constant regardless of molecular weight of polymer. Syndiotactic PVA gives a slope less than 1. The two PVA solutions

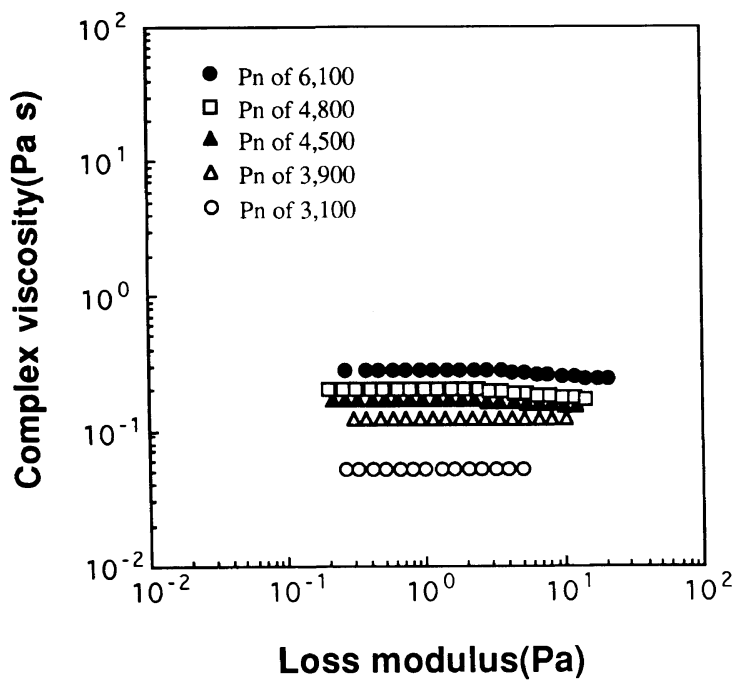

Figure 2. Viscosity curves of $2 \mathrm{wt} \%$ solutions of atactic PVA in DMSO at $30^{\circ} \mathrm{C}$.

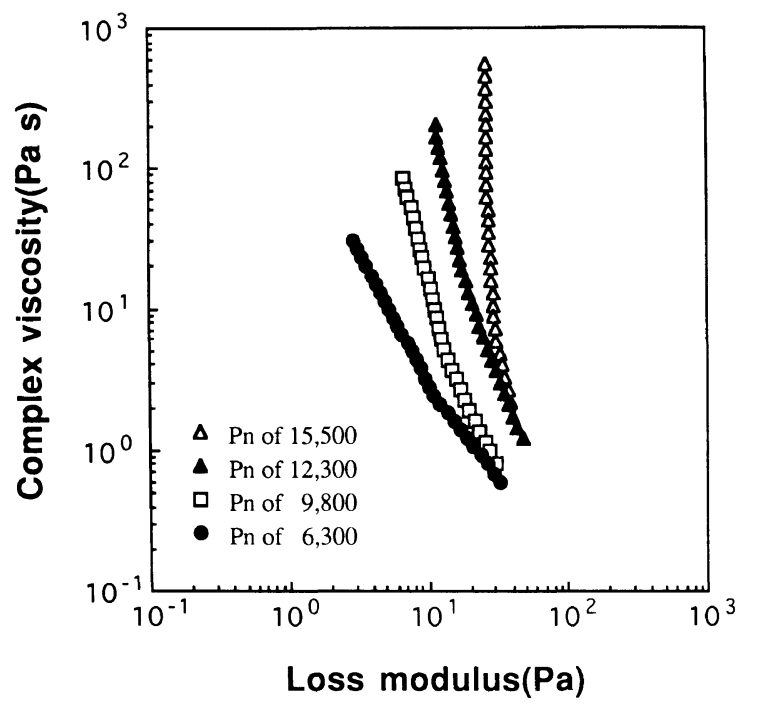

Figure 3. Viscosity curves of $2 \mathrm{wt} \%$ solutions of syndiotactic PVA in DMSO at $30^{\circ} \mathrm{C}$.

of identical polymer concentration containing polymers of similar molecular weight are comparable. Atactic PVA of $P_{n} 6100$ in Figure 4 and syndiotactic PVA of $P_{n} 6300$ in Figure 5. Atactic PVA solution gives a slope of 1 while syndiotactic PVA solution, of $c a$. 0.3 although the latter gives much greater $G^{\prime \prime}$ than the former at identical frequency. In these plots, the slopes represent increase in energy dissipation for that in shear rate. The ratio of $G^{\prime \prime}$ to $\omega$ would be constant if there is no internal structures formed by physical association of polymer molecules in solution, viz., if the individual polymer molecules are homogeneously and isotropically dissolved in solution. Thus the constant value of the slope 1 for atactic PVA irrespective of molecular weight of polymer is indicative of homogeneous isotropic solution. The energy dissipation per cycle would be reduced if internal structures exist in the solution because deformation of the structures consumes additional energy. Thus the smaller values of the slope of syndiotactic PVA in comparison with atactic PVA indicate that the former is more inhomogeneous than the latter. 


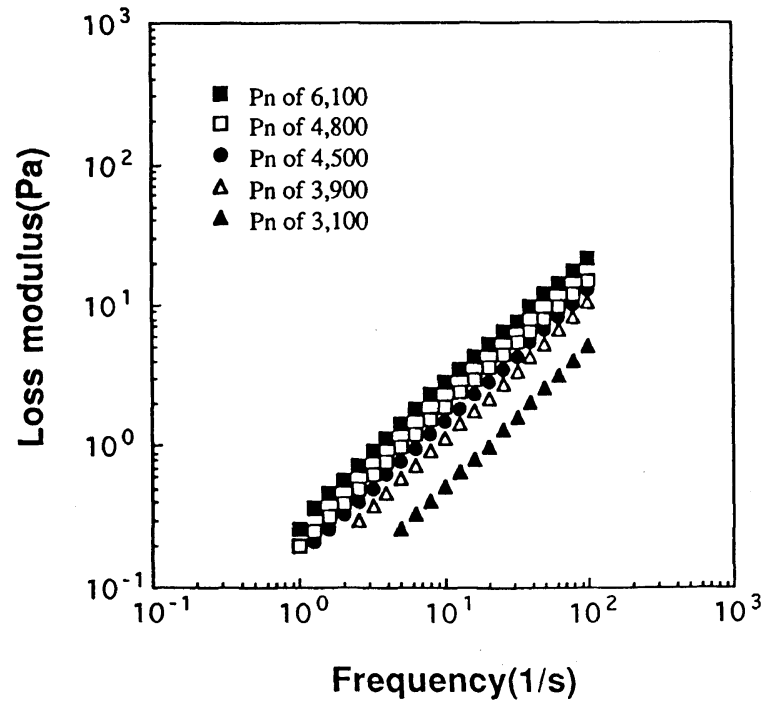

Figure 4. Plot of loss modulus vs. frequency for $2 \mathrm{wt} \%$ solutions of atactic PVA in DMSO at $30^{\circ} \mathrm{C}$.

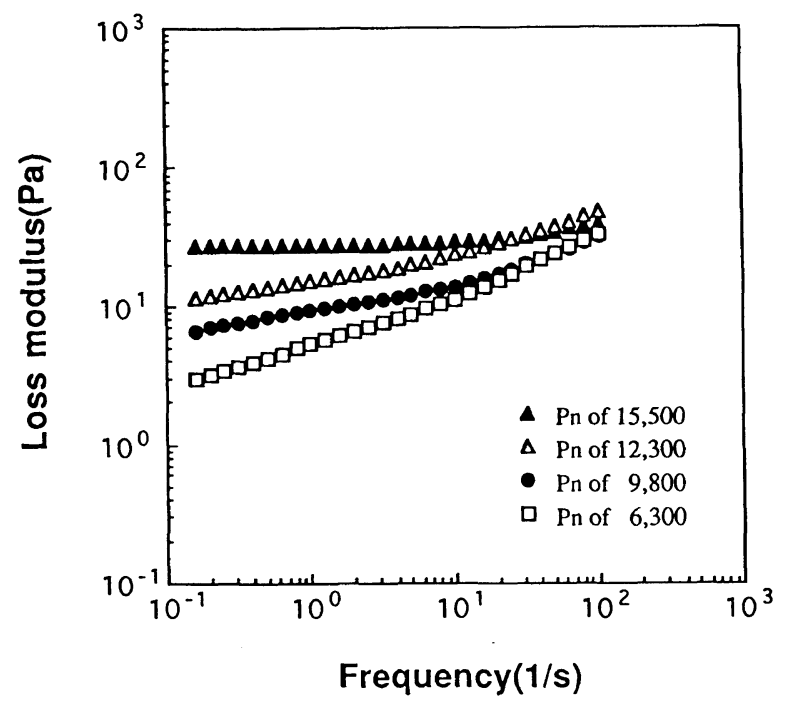

Figure 5. Plot of loss modulus $v s$. frequency for $2 \mathrm{wt} \%$ solutions of syndiotactic PVA in DMSO at $30^{\circ} \mathrm{C}$.

It should be also noted in Figure 5 that the slope of syndiotactic PVA decreases as the molecular weight of polymer increases, from 0.3 to almost zero as $P_{n}$ is increased from 6300 to 15500 . The decrease of the slope for syndiotactic PVA with increasing molecular weight of polymer implies that heterogeneity probably due to development of preorganizing domains with internal orders increases as molecular weight of polymer increases. The molecular weight effect suggests that syndiotactic PVA of higher molecular weight forms internal structures more readily. On the plot of storage modulus $\left(G^{\prime}\right)$ against $G^{\prime \prime}$, the slope of atactic PVA approaches 2 whereas that of syndiotactic PVA approaches $1 .{ }^{4}$ This further verifies that atactic PVA produces homogeneous isotropic solutions whereas syndiotactic PVA gives rise to heterogeneous solutions. ${ }^{18}$

Bingham flow behavior gives rise to a non-zero yield stress, which represents minimum energy required to break pseudostructures. The yield stress of heterogeneous systems can be determined from the Casson plot, plot of the square root of shear stress $(\tau)$ against square root

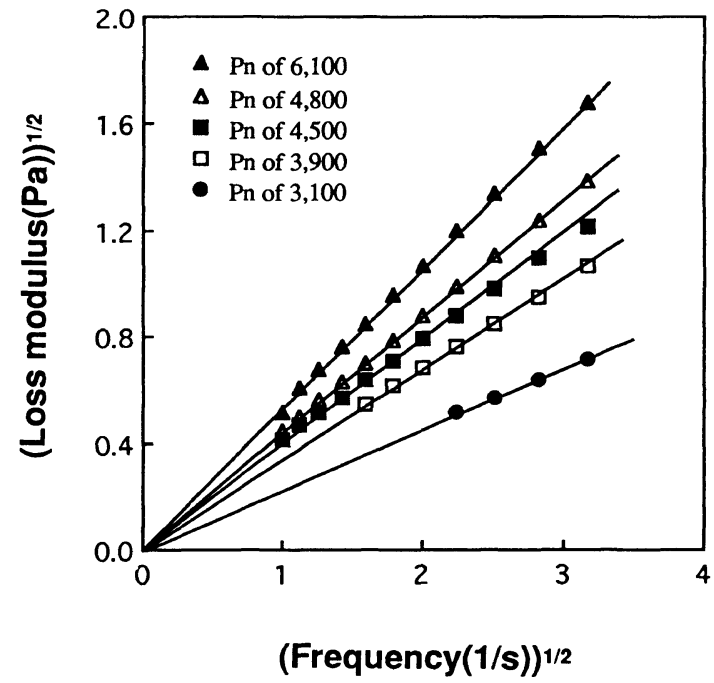

Figure 6. Plot of square root of loss modulus vs. square root of frequency for $2 \mathrm{wt} \%$ solutions of atactic PVA in DMSO at $30^{\circ} \mathrm{C}$.

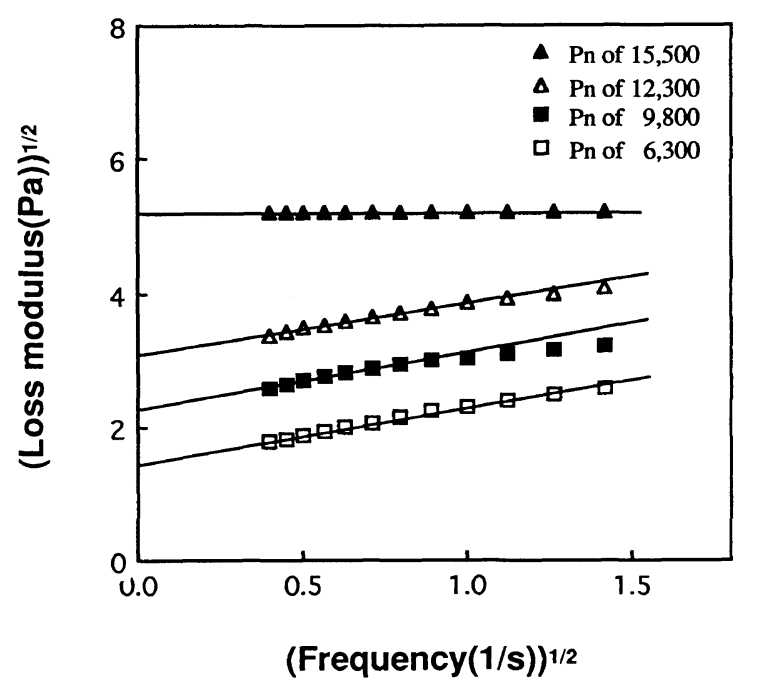

Figure 7. Plot of square root of loss modulus vs. square root of frequency for $2 \mathrm{wt} \%$ solutions of syndiotactic PVA in DMSO at $30^{\circ} \mathrm{C}$.

of shear rate $(\gamma)$ in steady shear flow. The yield stress $\left(\tau_{0}\right)$ is obtained from, ${ }^{19}$

$$
\tau^{1 / 2}=\tau_{0}{ }^{1 / 2}+K \gamma^{1 / 2}
$$

Similarly, the intercept of $G^{\prime \prime}$ axis $\left(G_{0}{ }^{\prime \prime}\right)$ on the plot of the square root of $G^{\prime \prime}$ against square root of frequency $(\omega)$ may be regarded as a measure of yield stress in dynamic shear measurement as, ${ }^{20}$

$$
G^{\prime 1 / 2}=G_{0}{ }^{1 / 2}+K^{\prime} \omega^{1 / 2}
$$

The square roots of $G^{\prime \prime}$ of $2 \mathrm{wt} \%$ solutions of atactic and syndiotactic (PVA)s of several different molecular weights in DMSO are plotted against the square root of $\omega$ in Figures 6 and 7, respectively. In the case of atactic PVA, all the intercepts reduce to zero in the so-called Casson-type plots whatever the molecular weight of polymer. This suggests that atactic PVA forms little pseudostructure in the DMSO solutions. Syndiotactic PVA produces non-zero intercepts on the Casson-type plot, pointing out the existence of yield stress of positive values. This suggests that some pseudostructure is 
present in solutions of syndiotactic PVA in DMSO. The yield stress notably increases with molecular weight of polymer at the identical polymer concentration in Figure 7.

The intermolecular interactions through polar hydroxyl groups may be more effective with syndiotactic PVA than atactic PVA since the hydroxyl groups are more regularly arranged along the molecule axis. ${ }^{21,22}$ Hence, syndiotactic PVA molecules may acquire greater chain stiffness in solution than atactic PVA molecules because the more regularly distributed hydroxyl groups make packing of the polymer chains more compact and more dense. $^{23}$ When syndiotactic PVA molecules have very high syndiotactic content, they may act like a rigid rod polymer. Thus, it stands to reason that the disorderorder transition results from increased stiffness of the polymer molecules. Dissolution of $2 \mathrm{wt} \%$ of syndiotactic PVA in DMSO produces something like a gel with extremely high viscosity while that of atactic PVA yields only a dilute solution of low viscosity.

Syndiotactic PVA has higher melting temperature $\left(T_{\mathrm{m}}\right)$ than atactic PVA even at similar molecular weights. In fact, thermal analysis by differential scanning calorimeter revealed that syndiotactic PVA with molecular weight of 7300 gave $T_{\mathrm{m}}$ of $248.5^{\circ} \mathrm{C}$ while atactic PVA with molecular weight of 7100 gave $T_{\mathrm{m}}$ of $232.8^{\circ} \mathrm{C} .^{13}$ This shows that syndiotactic PVA possesses higher chain stiffness than atactic PVA.

The phase morphology of 2 and $5 \mathrm{wt} \%$ solutions of atactic and syndiotactic (PVA)s in DMSO in the static (unsheared) and sheared states is shown in Figure 8. Even in the static state syndiotactic PVA solutions exhibit heterogeneous structures as shown in Figure 8(a). It is a little surprising to see that when the optically homogeneous solution of syndiotactic PVA in DMSO develops heterogeneous structures just by pouring onto a glass plate. The fibrillation of syndiotactic PVA molecules in the solution is more clearly visualized by the phase morphologies of the solution shown in Figure 8(a) after having sheared. As described in EXPERIMENTAL section, shear is exerted by slightly moving the upper glass in the direction of the plate at the velocity of around $2 \mathrm{~mm} \mathrm{~s}^{-1}$ for $0.5 \mathrm{~s}$. This may be explained as follows. First, preorganized structures have internal order which may be easily and readily oriented by elongation and shear exerted while pouring the solution. Secondly, phase separation results from shear-induced orientation and crystallization of polymer chains in the solutions. In fact, considering the unusual rheological response of solutions of syndiotactic PVA in DMSO the development of fibrillar structure on applying shear results from these two factors.

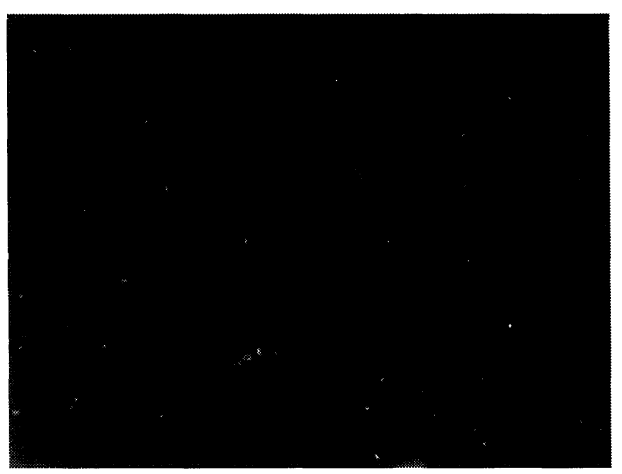

unsheared

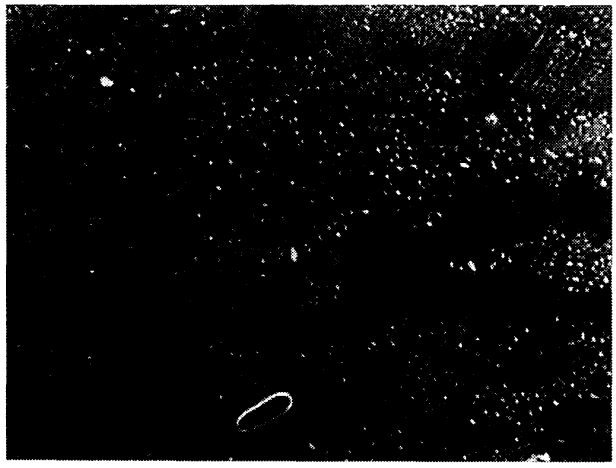

unsheared

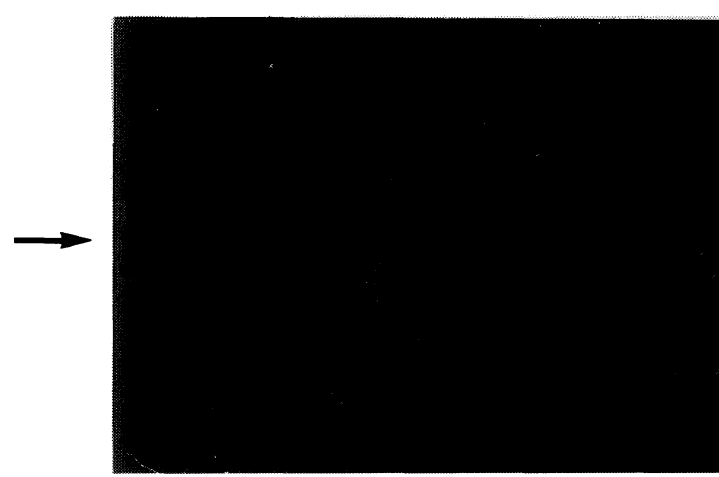

sheared

(a)

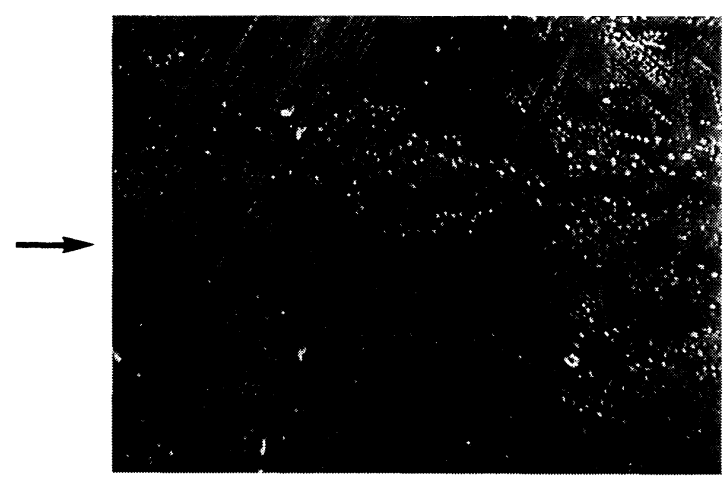

sheared

(b)

Figure 8. Static (unsheared) and sheared phase morphologies of $2 \mathrm{wt} \%$ solutions of PVA in DMSO at $30^{\circ} \mathrm{C}$ : (a) atactic PVA with $P_{n}$ of 6100 ; (b) syndiotactic PVA with $P_{n}$ of 6300 . 
In the case of 2 and $5 \mathrm{wt} \%$ solutions of atactic PVA in DMSO, heterogeneity by shear is not observed as shown in Figure 8(b). This shows that few heterogeneous structures develop in solution by shearing as typically observed in isotropic single phase solutions of ordinary flexible chain polymers.

In the case of syndiotactic PVA, increase of shear rate notably increases the flow birefringence at shear higher than $5 \mathrm{~s}^{-1}$, but gives rise to only a slight increase in the case of atactic PVA. ${ }^{4}$ This suggests that heterogeneity of syndiotactic PVA solutions results from the formation of preorganized domains with internal order. Preorganized domains were readily oriented by shear. It is not easy to imagine that the solution of flexible chain PVA includes such heterogeneous domains. However, repeated measurements of the rheological properties ascertain that the solution of syndiotactic PVA in DMSO contains heterogeneous domains. ${ }^{4}$

Investigation of relaxation behavior of atactic and syndiotactic (PVA)s may be of help to account for differences in the rheological properties of the two polymers. That is, relaxation time may be much longer

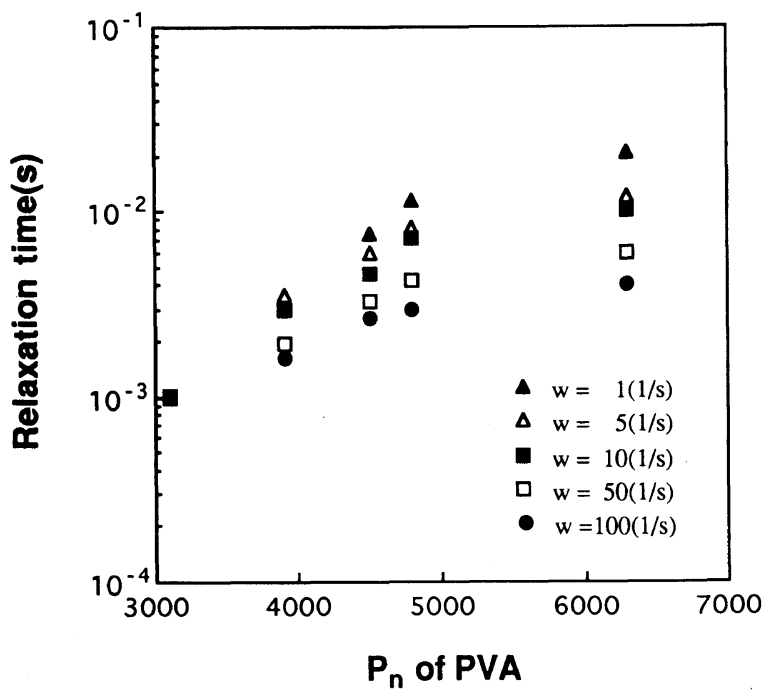

Figure 9. Variation in relaxation time of $2 \mathrm{wt} \%$ solutions of atactic PVA in DMSO at various frequencies at $30^{\circ} \mathrm{C}$.

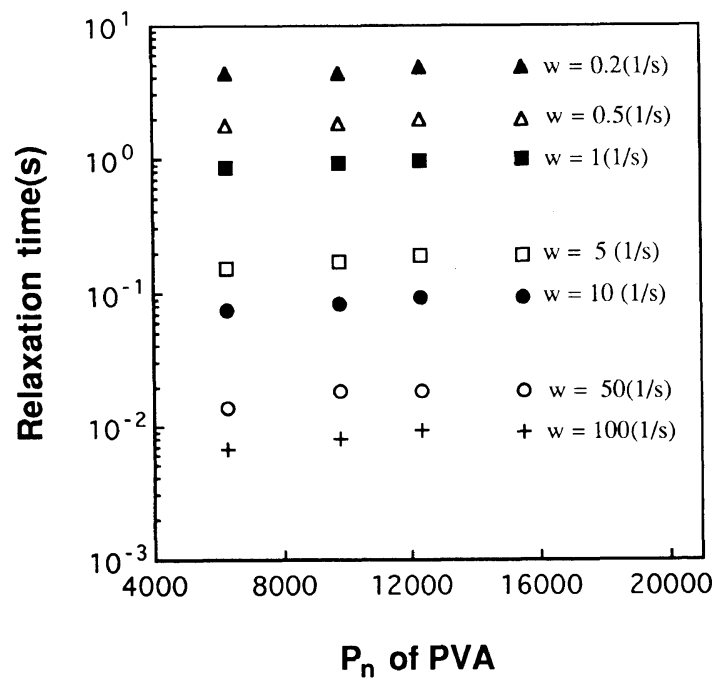

Figure 10. Variation in relaxation time of $2 \mathrm{wt} \%$ solutions of syndiotactic PVA in DMSO at various frequencies at $30^{\circ} \mathrm{C}$. if some molecular order exists. For instance, a rigid rod polymer exhibits very long relaxation time when it forms an anisotropic phase. Similarly, the formation of a mesophase also leads to long relaxation time. The relaxation time $(\lambda)$ under dynamic shear may be calculated as, $^{24}$

$$
J^{\prime}=\frac{G^{\prime}}{\left(\left[\eta^{*}\right] \omega\right)^{2}}=\frac{\lambda}{\left[\eta^{\prime}\right]}
$$

where $J^{\prime}$ and $G^{\prime}$ are compliance and storage modulus, respectively. Variation of relaxation time of $2 \mathrm{wt} \%$ solutions of atactic and syndiotactic (PVA)s in DMSO with frequency is shown in Figures 9 and 10. Syndiotactic PVA exhibits much longer relaxation time than atactic PVA. The relaxation time of atactic PVA notably increases with $P_{n}$ whereas that of syndiotactic PVA only slightly increases with $P_{n}$. The extent of reducing relaxation time with decreasing frequency is more in syndiotactic PVA than in atactic PVA. Atactic PVA molecules exhibit relaxation times typical of flexible chain polymers. However, it is surprising in the case of syndiotactic PVA that flexible chain polymer molecules exhibit such long relaxation times particularly at lower frequencies. Although the ratio of relaxation times of syndiotactic to atactic PVA decreases with frequency, the relaxation time of syndiotactic PVA is almost 100 times that of atactic PVA at $1 \mathrm{rad} \mathrm{s}^{-1}$ if one compares the two polymers with similar molecular weight. That relaxation time of syndiotactic PVA is almost independent of $P_{n}$ supports this as well.

To simulate actual in situ fibrillation during saponification, solutions of two syndiotactic (PVA)s with similar molecular weight but with different degrees of saponification, 95.4 and $99.0 \%$, have been prepared since in situ fiber formation is largely accomplished at the late stage of saponification of PVPi. ${ }^{13}$ Figure 11 shows plot of complex viscosity against $G^{\prime \prime}$ for the solutions of the two (PVA)s in DMSO. The slope of the viscosity curves demonstrates the effect of the degree of saponification on shear dependence of viscosity and yield behavior of

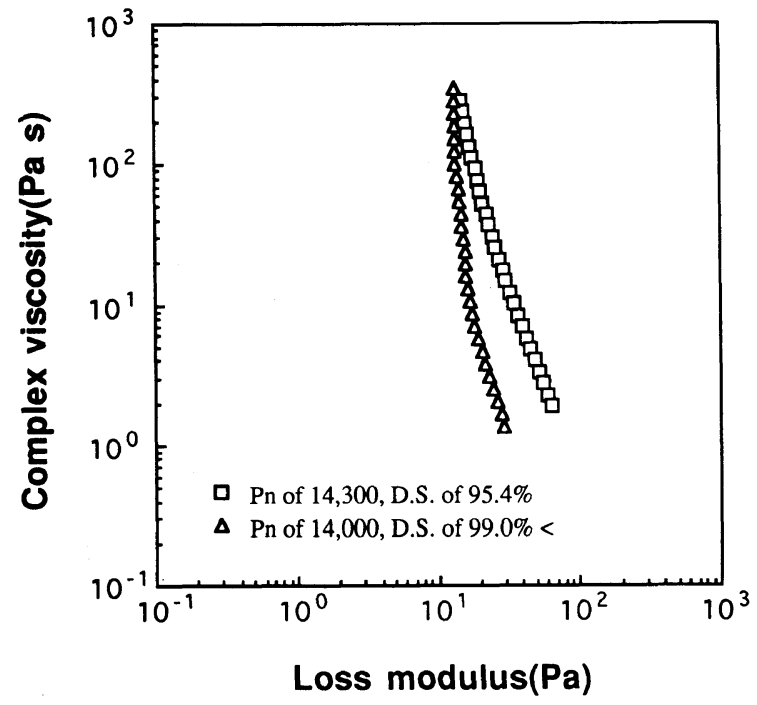

Figure 11. Effects of the degree of saponification of polymer on viscosity behavior of $2 \mathrm{wt} \%$ solutions of syndiotactic PVA in DMSO at $30^{\circ} \mathrm{C}$. 


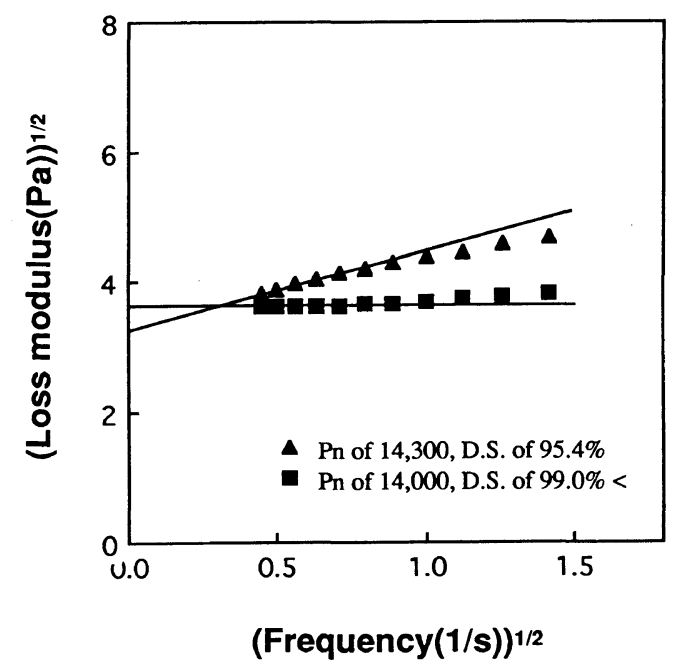

Figure 12. Effects of the degree of saponification of polymer on yield behavior of $2 \mathrm{wt} \%$ solutions of syndiotactic PVA in DMSO at $30^{\circ} \mathrm{C}$.

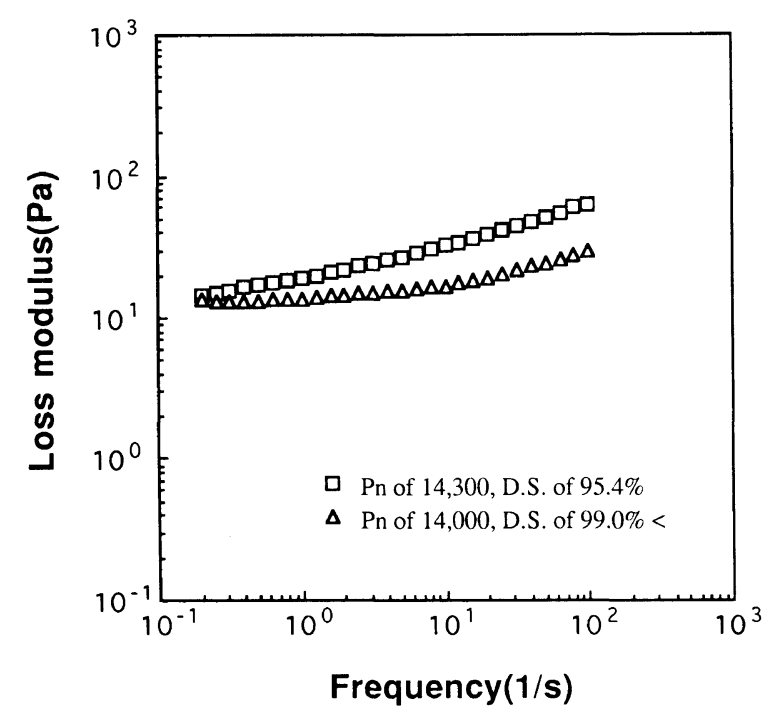

Figure 13. Effects of the degree of saponification of polymer on frequency dependence of loss modulus of $2 \mathrm{wt} \%$ solutions of syndiotactic PVA in DMSO at $30^{\circ} \mathrm{C}$.

syndiotactic PVA solutions. PVA with higher saponification shows more shear thinning at similar molecular weight of polymer, suggesting that PVA molecules are more readily oriented as saponification increases. This may provide indirect evidence of the spontaneous in situ orientation of polymer molecules at the late stage of saponification. As shown in Figure 12 both the syndiotactic (PVA)s exhibit yield behavior. Yield stress is higher for the polymer with higher degree of saponification at similar molecular weight of polymer. This indicates that more domains with internal order are produced at higher saponification, as confirmed by Figure 13. These facts result from increase in stiffness of polymer molecules with proceeding the saponification reaction.

\section{CONCLUSION}

The rheological characterization of solutions of (PVA)s with different syndiotactic diad content implied that there might exist a disorder-order transition when the syndiotactic diad content in PVA is higher than the critical value. PVA of syndiotactic content $52-53 \%$ exhibited rheological properties typical of most flexible chain polymers, whereas PVA of syndiotactic content $63-65 \%$ gave the rheological responses similar to the liquid crystalline polymers. The in situ development of highly crystalline PVA fibers with high degree of orientation without requiring additional drawing in the case of syndiotactic PVA further ascertained this. At this time, however, the precise value of the critical syndiotactic diad content at which the disorder-order transition takes place is not clear because it is too difficult to prepare (PVA)s with syndiotactic diad content covering the range of 53 to $63 \%$ in series.

\section{REFERENCES}

1. R. T. Morrison and R. N. Boyd, "Organic Chemistry," Allyn and Bacon, Inc., Boston, MA, 1973, p 261.

2. M. P. Stevens, "Polymer Chemistry," Oxford University Press, New York, N.Y., 1990, p 85.

3. G. Natta and P. Corradini, J. Polym. Sci., 20, 251 (1956).

4. W. S. Lyoo, Ph.D. Thesis, Seoul National University, 1994.

5. M. P. Stevens, "Polymer Chemistry," Oxford University Press, New York, N.Y., 1990, pp 209-212.

6. K. Yamamura, I. Mizutani, K. Monma, T. Tanigami, and S. Matsuzawa, J. Appl. Polym. Sci., 31, 521 (1986).

7. I. Sakurada and N. Fujiwara, Kobunshi Kagaku, 2, 143 (1945).

8. T. Yamamoto, S. Seki, R. Fukae, O. Sangen, and M. Kamachi, Polym. J., 22, 567 (1990).

9. N. Nakajima, Kobunshi Kagaku, 11, 142 (1954).

10. R. Lanthier, U.S. Patent 3,303,174 (1967).

11. T. C. Wu and J. C. West, U.S. Patent 4,463,138 (1982).

12. B. C. Kim, in "Polymeric Materials Encyclopedia," J. C. Salamone, Ed., CRC Press Inc., Boca Raton, FL, 1996, pp $2350-2359$.

13. W. S. Lyoo, Ph. D. Thesis, Seoul National University (1994).

14. S. Ottani, A. Valenza, and F. P. La Mantia, Rheol. Acta, 27, 172 (1988).

15. T. M. Malik, P. J. Carreau, and N. Chapleau, Polym. Eng. Sci., 29, 600 (1989).

16. A. I. Isayev and M. Modic, Polym. Compos., 8, 158 (1987).

17. F. P. La Mantia, A. Valenza, M. Paci, and P. L. Magagnini, Rheol. Acta, 28, 417 (1989).

18. S. S. Kim and C. D. Han, Macromolecules, 26, 6633 (1993).

19. N. Casson, in "Rheology of Dispersed Systems," C. C. Mill, Ed., Pergamon, London, 1959.

20. S. M. Hong, B. C. Kim, S. S. Hwang, and K. U. Kim, Polym. Eng. Sci., 33, 630 (1993).

21. C. W. Bunn and H. S. Peiser, Nature, 161, 159 (1947).

22. C. W. Bunn, Nature, 161, 929 (1948).

23. M. Nagura, S. Matsuzawa, K. Yamamura, and H. Ishikawa, Polym. Commun., 24, 250 (1983).

24. K. F. Wissbrun and A. C. Griffin, J. Polym. Sci., Polym. Phys. Ed., 20, 1835 (1982). 